\title{
Polichotomies on Imbalanced Domains by One-per-Class Compensated Reconstruction Rule
}

\author{
Roberto D'Ambrosio and Paolo Soda \\ Integrated Research Centre, Universitá Campus Bio-Medico of Rome, Rome, Italy \\ $\{r$.dambrosio, p. soda\}@unicampus.it
}

\begin{abstract}
A key issue in machine learning is the ability to cope with recognition problems where one or more classes are under-represented with respect to the others. Indeed, traditional algorithms fail under class imbalanced distribution resulting in low predictive accuracy over the minority classes. While large literature exists on binary imbalanced tasks, few researches exist for multiclass learning. In this respect, we present here a new method for imbalanced multiclass learning within the One-per-Class decomposition framework. Once the multiclass task is divided into several binary tasks, the proposed reconstruction rule discriminates between safe and dangerous classifications. Then, it sets the multiclass label using information on both data distributions and classification reliabilities provided by each binary classifier, lowering the effects of class skew and improving the performance. We favorably compare the proposed reconstruction rule with the standard One-per-Class method on ten datasets using four classifiers.
\end{abstract}

\section{Introduction}

In data mining and machine learning, we deal with imbalanced (or skewed) recognition problem when one of the classes is largely under-represented in comparison to the others. Most traditional learning algorithms cannot cope with this case since they are biased towards the majority classes, resulting in poor predictive accuracy over the minority ones. This happens because they are designed to minimize errors over training samples, ignoring classes composed of few instances.

In real world applications, such as text classification, currency validation, and medical diagnosis, very often the a priori distributions of samples are different among the classes, thus resulting in an imbalanced classification task. Due to the relevance of the topic and its potential impact on the development of learning algorithms, in the recent years there have been several works proposing learning methods coping with skewed training set (TS). In particular, most of the existing literature focus on class imbalance learning methods for binary problems (also referred to as dichotomies), proposing solutions both at data and algorithmic levels, e.g. [1, 2]. Approaches working at data level provide different forms of resampling, e.g. random or direct oversampling and undersampling, whereas those working at algorithmic level introduce a bias to compensate the skewness of the classes, e.g. adjusting the costs of classes, adjusting decision thresholds and recognition-based learning.

Otherwise, learning under a skewed multiclass TS has received little attention despite the fact that such problems can be found in a large number of domains. The few recent 
works focused on multiclass learning (also named as polichotomy) can be roughly divided into two categories [3-7].

The first compensates class imbalance directly on the polychotomy [3, 5, 7]. In [5] the authors present a two-stage evolutionary neural network algorithm with the entropy and area fitness functions, under the assumption that a good classifier should combine a high classification rate level in the global dataset with an acceptable level for each class. In [7] the authors propose a small sphere and large margin approach for novelty detection problems, where the majority of training data are normal examples, and the training data also contain a small number of abnormal examples or outliers. Their basic idea is to construct a hypersphere solving a convex optimization problem containing most of the normal examples, such that the volume of this sphere is as small as possible, while at the same time the margin between the surface of this sphere and the outlier training data is as large as possible. In [3] the authors introducing several cost functions in the learning algorithm of a neural network in order to improve its generalization ability and speed up the convergence process.

The second category faces with polychotomy using a decomposition approach [4, 6], which consists in reducing the multiclass problem complexity in less complex binary subtasks, each one addressed by a classifier usually referred to as dichotomizer [8-11]. To provide the final classification, dichotomizers' outputs are combined according to a reconstruction rule. We found only two attempts proposing reconstruction rule suited for imbalance datasets [4, 6]. In particular, in [4] the authors applied the SMOTE algorithm [1], i.e. an oversampling method for binary skewed task, in each dichotomizer and then combine their outputs via a fuzzy model. In [6] the authors applied in the learning phase of each dichotomizer several methods compensating binary class imbalance.

The short analysis of the literature reported so far shows that multiclass learning in class imbalance circumstances is an issue deserving more research efforts. In this respect, we propose here a new reconstruction rule combining dichotomizers' outputs within the One-per-Class decomposition framework, thus falling into the second of the aforementioned branch. Once distinguished between safe and dangerous dichotomizers classifications on the basis of sample classification reliability, it applies different reconstruction rules for each of these two cases thus permitting to reduce effects due to the skewness between classes. We test our approach on ten databases using four different classification architectures, and we compare its results with those provided both by a well known One-per-Class reconstruction rule and by a multiclass classifier.

\section{Background}

In this section we first introduce decomposition methods with particular reference to the One-per-Class approach, and then we discuss performance metrics suited for class imbalance classification tasks.

\subsection{One-per-Class Decomposition Method}

Decomposition methods reduce multiclass problem complexity in less complex dichotomies, each one addressed by a classifier. Their rationale lies in observing that, on 
the one hand, discriminating between two classes is much easier than simultaneously distinguishing among many classes [12] and, on the other hand, most of the available classification algorithms are best suited to learn binary functions [13, 14].

Decomposition methods can be unified in a common framework wherein the output space is represented by a binary code matrix named as decomposition matrix. On its basis, it is then possible to distinguish between the different approaches as follows. Let $\Omega=\left\{\omega_{1}, \omega_{2}, \ldots, \omega_{K}\right\}$ represents the label set of the $K$-classes problem, with $K>2$.

The decomposition of the polychotomy generates a pool of $L$ dichotomizers, with the value of $L$ depending upon the decomposition approach adopted. The dichotomizer $M_{i}$ is a discriminating function that classifies each input sample in two separate superclasses, represented by the label set $\Omega_{i}=\{-1,1\}$, each label identifying a subset of polychotomy classes. Therefore, the overall decomposition scheme can be set by a decomposition matrix $D \in \Re^{L} \mathrm{x} \Re^{c}$, whose elements are defined as:

$$
d_{i j}=\left\{\begin{array}{l}
1 \quad \text { if class } j \text { is in the subgroup associated to label } 1 \text { of } M_{i} \\
-1 \text { if class } j \text { is in the subgroup associated to label }-1 \text { of } M_{i} \\
0 \quad \text { if class } j \text { is in neither groups associated to label }-1 \text { or } 1 \text { of } M_{i}
\end{array}\right.
$$

Hence, the dichotomizer $M_{i}$ is trained to associate patterns belonging to class $\omega_{j}$ with values $d_{i j}$. Within this framework, the main decomposition approaches are named as One-per-Class (OpC) [8, 11], Error-Correcting Output Code [8, 9], and PairWise Coupling [10]. In the following we further present the OpC approach for four reasons. First, it is applied in the reconstruction rule proposed in this paper; second, it introduces a strong degree of imbalance for each dichotomizer since it collapses samples of all classes except one into a superclass; third, it is a very popular method with the lowest computational complexity and, fourth, it is very often used to derive multiclass classifier from learning algorithms which are intrinsically binary.

OpC reduces the multiclass problems into $K$ binary problems (i.e. $L=K$ ), each one addressed by one dichotomizer, thus achieving a squared decomposition matrix. We say that the $j$ th dichotomizer is specialized in the $j$ th class when it aims at recognizing if the input sample belongs either to the $j$ th class or, alternatively, to any other class. Without loss of generality and to simplify the notation we use label 0 instead of -1 . Therefore, the binary label of the $j$ th dichotomizer is 1 if the sample $x$ belongs to the $j$ th class, and 0 otherwise.

It is worth noting that dichotomizers, besides labelling each pattern, may supply other information typically related to the degree that the sample belongs (or does not belong) to the corresponding class. Indeed, it has been proved that exploiting information derived from classifiers working at the measurement level permits to define reconstruction rules that are potentially more effective [15]. Since measurement classifiers can provide more information than other classifiers, we assume that only measurement experts are used in $\mathrm{OpC}$ scheme. This assumption is not a limitation since it is always possible to obtain a measurement for each classification act of any kind of classifiers [16]. 


\subsection{Performance Metrics}

The confusion matrix is usually used to assess the performance of a recognition system permitting to compute several indexes. The most used one is the global recognition accuracy $(a c c)$ defined as $a c c=\frac{\sum_{j=1}^{K} n_{j j}}{N}$, where $n_{j j}$ is the number of elements of class $j$ correctly labelled and $N$ is the total number of samples.

However, in case of imbalanced TS the recognition performance cannot be measured in terms of classification accuracy only, since this measure is strongly biased to favor the majority class. Hence, it would be more interesting to use a performance measure dissociating the hits (or the errors) that occur in each class. To this aim, we can compute the accuracy by class as $a c c_{j}=\frac{n_{j j}}{N_{j}}$, where $N_{j}$ is the number of samples in the $j$-th class. Notice that $a c c_{j}$ is independent of prior probabilities and, thus, it is robust when class distribution might be different in training and test sets or change over time. From such metrics we can compute an overall index by extending the geometric mean of accuracies $(g)$ typically used to assess the performance in binary skewed problems. It is given by $g=\left(\prod_{j=1}^{K} a c c_{j}\right)^{\frac{1}{K}}$, being a non-linear measure since a change in one of its arguments has a different effect on $g$ depending on its magnitude. For instance, if a classifier misses the labels of all samples in the $j$ th class, it results $a c c_{j}=0$, and $g=0$.

\section{Compensated Reconstruction Rule}

In this section we propose a reconstruction rule suited for $\mathrm{OpC}$ scheme that, combining dichotomizers' outputs, reduces drawbacks given by learning under data skewness and improves system performance. Indeed, the typical error rate minimisation performed by most of learning algorithms over the TS introduces a bias in favour of the the majority class, resulting in low accuracies on classes composed of few instances. Hereinafter, the proposed rule is referred to as Compensated Reconstruction Rule (CRR).

On the basis of the outputs provided by the pool of dichotomizers we distinguish between safe and dangerous classifications. Safe classifications are those in which each dichotomizer is strongly confident about its output. Dangerous classifications are those in which two or more dichotomizers are weakly confident about their predictions. Considering the skewed nature of the dataset, the low confidence of two or more dichotomizers with their outputs increases the likelihood of providing wrong classification. The confidence of a classifier on its output should be measured by using classification reliability, i.e. a measure lying in $[0,1]$ computed by any measurement classifier [16, 17]. Now, let us introduce the following notation:

- $\Omega=\left\{\omega_{1}, \omega_{2}, \ldots, \omega_{K}\right\}$ is the set of class labels, as reported in section 2.1,

- $N$ is the total number of samples as reported in section 2.2.

- $N_{j}$ is the number of samples belonging to the class $\omega_{j}$, as reported in section 2.2,

- $x \in \Re^{n}$ is a sample;

- the binary profile $\mathbf{M}(x)$ is the $K$-bit codeword of $x$ collecting dichotomizers' outputs as $\mathbf{M}(x)=\left[M_{1}(x), M_{2}(x), \ldots, M_{K}(x)\right] . M_{j}(x)$ is 1 if $x \in \omega_{j}, 0$ otherwise;

- the reliability profile $\boldsymbol{\Psi}(x)$ is a $K$ elements vector collecting dichotomizers' reliabilities; each entry measures the reliability of the $j$ th dichotomizer's output and represents the degree that $x$ belong or not to predicted class. It is given by $\boldsymbol{\Psi}(x)=$ $\left[\psi_{1}(x), \psi_{2}(x), \ldots, \psi_{K}(x)\right]$; 
- the reverse a-priori probability profile $\mathbf{R}$ contains the knowledge on the a-priori classes distribution; it is a $K$ elements vector $\mathbf{R}=\left[r_{1}, r_{2}, \ldots, r_{K}\right]$, where $r_{j}=$ $1-N_{j} / N$

- $\tau_{0}$ is a threshold for detecting classifications more likely to be corrected in case of dichotomizer suggesting that $x$ does not belong to its class $\left(M_{j}(x)=0\right)$. Its value is estimated on a validation set maximizing $a c c_{j}$;

- $\tau_{1}$ is a threshold for detecting classifications more likely to be corrected in case of dichotomizer suggesting that $x$ belongs to its class $\left(M_{j}(x)=1\right)$. Its value is set as for $\tau_{0}$.

On this basis, the classification of $x$ is said to be a safe classification if:

$$
\left(\alpha_{1}(x)>\tau_{1} \wedge \alpha_{0}(x)>\tau_{0}\right) \vee\left(\alpha_{1}(x)>\tau_{1} \wedge \alpha_{0}(x)<\tau_{0} \wedge \overline{\alpha_{0}}(x)>\tau_{0}\right)
$$

where $\alpha_{1}(x)=\max _{j}\left(\psi_{j}(x) \mid M_{j}(x)=1\right)$ is the largest value of reliability among those provided by dichotomizers whose outputs is 1, i.e. the dichotomizers suggesting that $x$ belongs to their corresponding class. $\alpha_{0}(x)=\min _{j}\left(\psi_{j}(x) \mid M_{j}(x)=0\right)$ is the lowest reliability value among those provided by dichotomizers whose outputs are 0 , i.e. the dichotomizers suggesting that $x$ does not belong to their corresponding class. Furthermore, $\overline{\alpha_{0}}(x)=E\left(\psi_{j}(x) \mid M_{j}(x)=0\right)$ is the average value of reliabilities associated with dichotomizers whose outputs are 0 . In the following, for brevity we omit to indicate that $\alpha_{0}, \alpha_{1}$ and $\overline{\alpha_{0}}$ depend on $(x)$ when this does not introduce any ambiguity.

The classification of $x$ is defined as dangerous when:

$$
\left(\alpha_{1}<\tau_{1} \wedge \alpha_{0}<\tau_{0}\right) \vee\left(\alpha_{1}>\tau_{1} \wedge \alpha_{0}<\tau_{0} \wedge \overline{\alpha_{0}}<\tau_{0}\right)
$$

To set the final classification, CRR applies different criteria for safe and dangerous classifications. In the former case, the index $s$ of the dichotomizer setting the final class $\omega_{s} \in \Omega$ is given by:

$$
s=\left\{\begin{array}{l}
\operatorname{argmax}_{j}\left(M_{j}(x) \cdot \psi_{j}(x)\right) \text { if } m \in[1, K] \\
\operatorname{argmim}_{j}\left(\overline{M_{j}(x)} \cdot \psi_{j}(x)\right) \text { if } m=0
\end{array}\right.
$$

where $\overline{M_{j}(x)}$ is the negate output of the dichotomizer and $m=\sum_{j=1}^{K} M_{j}(x)$. Notice that in such a case the final decision depends on both $\mathbf{M}(x)$ and $\boldsymbol{\Psi}(x)$ without considering data related to the degree of imbalance presented in the dataset.

In case of dangerous classification, when an error due to class skew is more likely to occur, we advantage the minority class in order to compensate classifier bias. To this aim, we exploit information contained in $\mathbf{R}$, whereas information provided by $\mathbf{M}(x)$ and $\boldsymbol{\Psi}(x)$ has been used to detected the dangerous situation itself. To set the final decision we consider only two dichotomizers. The first is the dichotomizer which more likely suggests that $x$ belongs to its class, since its output is one and it has the largest reliability among the others providing the same output. The second is the dichotomizer which more likely suggests that $x$ does not belong to class $j$ where the $j$ th dichotomizer is specialized on, but $x$ should belong to class $i$, with $j \neq i$. Indeed, the output $M_{j}(x)$ of this dichotomizer is zero and it has the lowest reliability among the others providing the same output (i.e. $\alpha_{0}$ ). The CRR sets the index $s$ as follows:

$$
s= \begin{cases}\operatorname{argmax}_{j}\left(r_{j}^{0}, r_{j}^{1}\right) & \text { if } \alpha_{1} \geq \alpha_{0} \\ \operatorname{argmim}_{j}\left(r_{j}^{0}, r_{j}^{1}\right) & \text { if } \alpha_{1}<\alpha_{0}\end{cases}
$$


where

$$
r_{j}^{i}=\left(r_{j} \mid M_{j}(x)=i \wedge \psi_{j}=\alpha_{i}\right), \text { with } i=\{0,1\}
$$

To explain the rationale of eq. 5, recall that we are considering dangerous classification where an error due to class skew is more likely to occur. Indeed, when $\alpha_{1} \geq \alpha_{0}$ and taking into account eq. 3. two cases may happen. In the first one, values of both $\alpha_{0}$ and $\alpha_{1}$ should be below the thresholds $\tau_{0}$ and $\tau_{1}$ respectively. In the second one, $\alpha_{1}$ is large but the average reliability $\overline{\alpha_{0}}$ of dichotomizers suggesting that the $x$ does not belong to their class $\left(M_{j}(x)=0\right)$ is below a threshold $\tau_{0}$. In both cases, it is reasonable to assume that wrong classification is given by the bias in favor of majority class; hence, CRR sets the final label as the class where the dichotomizer with the largest value of the reverse a-priori probability is specialized, i.e. the minority class among those considered.

Ortherwise, when $\alpha_{1}<\alpha_{0}$, CRR sets the final class as the one where the dichotomizer with the lowest reverse a-priori probability is specialized. In this case, considering again that this is a dangerous classification, $\alpha_{0}$ is smaller than threshold $\tau_{0}$ (eq. 3), and $\alpha_{1}$ is smaller than $\tau_{1}$ but it is smaller than $\alpha_{0}$ (eq. 5) . This observation implies that reliabilities values are small and noisy, suggesting that there isn't a clear trend among the dichotomizer outputs to set the final decision: hence, the class with the largest a-priori probability should be preferred to set the final classification.

Table 1. Summary of the used datasets. Symbols ${ }^{*}$ and ${ }^{+}$marks public and private datasets, respectively

\begin{tabular}{llllccccccc}
\hline \hline & \multicolumn{1}{c}{ Dataset } \\
${$\cline { 2 - 6 }$^{*}} }$ & GLASS $^{*}$ & ISOLET $^{*}$ & LETTER $^{*}$ & OPTDIGIT $^{*}$ & IIFI $^{*}$ & IIFH2 $^{+}$ & SAT $^{*}$ & WFRN $^{*}$ & WINE* $^{*}$ \\
N. of samples & 876 & 205 & 2000 & 2561 & 5620 & 600 & 573 & 6425 & 5456 & 178 \\
N. of classes & 6 & 5 & 26 & 26 & 10 & 3 & 5 & 6 & 4 & 3 \\
N. of features & 50 & 9 & 30 & 16 & 60 & 57 & 159 & 36 & 24 & 13 \\
Majority class & $28.1 \%$ & $37.0 \%$ & $4.7 \%$ & $4.5 \%$ & $10.2 \%$ & $36.0 \%$ & $37.0 \%$ & $23.9 \%$ & $40.4 \%$ & $39.9 \%$ \\
Minority class & $7.5 \%$ & $6.3 \%$ & $3.1 \%$ & $2.9 \%$ & $9.8 \%$ & $31.5 \%$ & $8.2 \%$ & $9.7 \%$ & $6.0 \%$ & $27.0 \%$ \\
\hline \hline
\end{tabular}

\section{Experimental Evaluation}

The method proposed in this paper has been compared to both a multiclass classifier and a well established $\mathrm{OpC}$ reconstruction rule, namely the Hamming decoding (HAMDEC) [8]. According to its original definition and assuming that each dichotomizer provides 1 or -1 labels, the index $s$ of the dicothomizer setting the final class $\omega_{s} \in \Omega$ is given by:

$$
s=\operatorname{argmin}_{i} d_{H}\left(\mathbf{D}\left(\omega_{i}\right), \mathbf{M}(x)\right)
$$

where

$$
d_{H}\left(\mathbf{D}\left(\omega_{i}\right), \mathbf{M}(x)\right)=\sum_{j=1}^{K}\left(\frac{1-\operatorname{sign}\left(D\left(\omega_{i}, j\right) M_{j}(x)\right)}{2}\right)
$$

The experiments have been carried out by employing three different paradigms for the base dichotomizers: a k-Nearest Neighbor $(\mathrm{kNN})$ as a statistical classifier, a Multi-Layer Perceptron (MLP) as a neural network, and a Support Vector Machine (SVM) as a 
kernel machine. With reference to multiclass classifier, we have considered the kNN and MLP network. Note that we have not considered multiclass SVM since it is usually implemented using a decomposition approach.

In particular, we used a kNN classifier choosing the $k$ value within the range $\{1,3,5,7\}$ that optimize performance on five validation set. To evaluate the reliability of kNN decisions we adopted a method that estimates the test patterns credibility on the basis of their quality in the feature space [17].

For the MLP, the neural network has a number of hidden layers equal to half of the sum of features number plus class number. The number of neurons is given in the input layer by the number of the features, whereas in the output layer is equal to the number of the classes. As with the kNN classifier, to evaluate the reliability of MLP decisions we adopted the method reported in [17].

In case of SVM we used both a SVMs with a Gaussian Radial Basis Function (RBF) and linear kernels, denoted as $\mathbf{S V M}_{r b f}$ and $\mathrm{SVM}_{l}$, respectively. For $\mathbf{S V M}_{r b f}$, the values of regularization parameter $C$ and scaling factor $\sigma$ have been selected within $\left\{1,10, \ldots, 10^{4}\right\}$ and $\left\{10^{-4}, 10^{-3}, \ldots, 10\right\}$, respectively. For $\mathrm{SVM}_{l}$, the values of cost $C$ is selected within $\left\{2^{ \pm 4}, 2^{ \pm 3}, 2^{ \pm 2}, 2^{ \pm 1}, 2^{0}\right\}$. Each parameter value is selected according to average performance of classifier on five validation set. The reliability of a SVM classification is estimated as proposed in [18].

In our experiments, we used nine public and one private datasets. These datasets are characterized by a large variability in the number of features, classes and samples, allowing the assessment of the performance in different conditions (Table 1). They are: Facial Expression recognition (FER) [19, 20], Indirect Immunofluorescence Intensity (IIFI) [21], Indirect Immunofluorescence HEp-2 cells staining pattern (IIFH2) [22], Glass Identification (GLASS) [23], ISOLET [23], Letter Recognition (LETTER) [23], Optical Recognition of Handwritten Digits Data Set (OPTDIGIT) [23], Statlog (SAT) [23], Wall Following Robot Navigation Data (WFRN) [23], and Wine (WINE) [23]. For every dataset classification accuracy $a c c$ and geometric mean of accuracies $g$ are computed averaging out values obtained performing ten fold cross validation. Each fold is computed maintaining the a-priori distribution of data showed by by original TS.

Results reported in Table 2 permit us to compare performance of CRR-based classification with those achieved both by a multiclass approach and a popular OpC scheme, employing also different classification paradigms.

With regard to the comparison between the CRR approach and the multiclass classification we observe that, generally, the first performs better than the latter both in term of $a c c$ and $g$. Indeed, the kNN with the CRR approach achieves better results than the corresponding multiclass scheme on all the tested datasets. In case of MLP neural network, the CRR scheme outperforms the multiclass classifier in eight cases out of ten datasets. In both cases $a c c$ and $g$ values improvements are up to $88 \%$.

Let us now focusing our attention to the comparisons between CRR and HAMDEC reconstruction schemes. With reference to the kNN classification paradigm, we notice that CRR outperforms the HAMDEC scheme in nine out of ten datasets both in terms of $a c c$ and $g$,showing improvements up to $18.0 \%$ and $14.0 \%$, respectively. Exploring MLP results in terms of $a c c$, we observe that CRR scheme outperforms HAMDEC in eight case out of ten, with the improvements ranging between from $1.1 \%$ up to $8.7 \%$. In case of 
Table 2. MLP, kNN and SVM performance ( $a c c \%$ and $g \%$ values) on ten public datasets

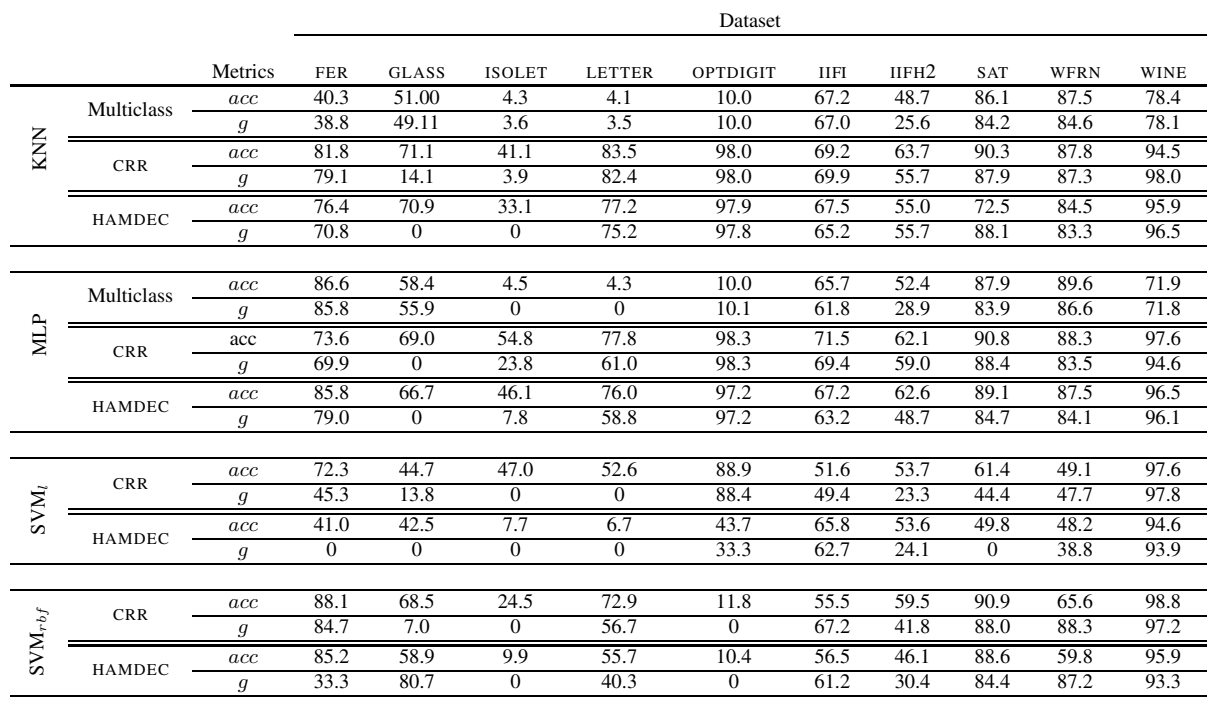

$g$ values, we observe that CRR has performance improvement up to $16.0 \%$ in seven case out of ten. SVM architecture employing CRR scheme shows larger accuracy than the one adopting HAMDEC scheme, independently of the used kernel. In particular, in nine out of ten datasets $\mathrm{SVM}_{l}$ and $\mathrm{SVM}_{r b f}$ improvements are up to $46 \%$ and 17\%, respectively. In term of $g$ and using $\mathrm{SVM}_{r b f}$ CRR scheme outperforms HAMDEC in eight out of ten cases with improvements up to 55\%. Using $\mathrm{SVM}_{r b f}$ improvements concern nine out of ten datasets, with the $g$ value raising up to $16 \%$.

Before concluding the paper, let us introduce two further considerations on the results measured in terms of $g$. First, it is worth noting that $g$ improvements are large (up to $55 \%$ ), especially when the base classifier is the $\mathrm{SVM}_{l}$. Second, in several cases where the application of the HAMDEC reconstruction rules provides values of $g$ equal to zero, which correspond to misclassifying all samples of one or more minority classes, the CRR rule attains larger and non zero $g$ values.

These last observations together with the results previously discussed point out that CRR scheme provides larger relative accuracies among all classes, thus achieving better classifications on classes with few samples and reducing class imbalance effects.

\section{Conclusion}

In this paper we presented a reconstruction rule for the One-per-Class decomposition framework overcoming issues related to learning under class skew in a multiclass scenario. It applies different criteria to set the final decision on the basis of both the outputs of the dichotomizers and the a-priori probabilities of the classes. The approach has been tested on ten datasets and successfully compared against a multiclass classifier and a well established $\mathrm{OpC}$ reconstruction rule. 


\section{References}

1. Chawla, N.V., et al.: SMOTE: Synthetic minority over-sampling technique. J. of Artificial Intelligence Research 16(3), 321-357 (2002)

2. Soda, P.: A multi-objective optimisation approach for class-imbalance learning. Pattern Recognition 44, 1801-1810 (2011)

3. Alejo, R., et al.: An empirical study for the multi-class imbalance problem with neural networks. Progress in Pattern Recog., Image Analysis and Applications, 479-486 (2008)

4. Fernández, et al.: Multi-class imbalanced data-sets with linguistic fuzzy rule based classification systems based on pairwise learning. Comp. Intel. for Knowledge-Based Systems Design, 89-98 (2010)

5. Martínez-Estudillo, F.J., et al.: Evolutionary learning by a sensitivity-accuracy approach for multi-class problems. In: IEEE WCCI, pp. 1581-1588 (2008)

6. Soda, P., et al.: Decomposition methods and learning approaches for imbalanced dataset: An experimental integration. In: 20th Int. Conf. on Pattern Recognition, pp. 3117-3120 (2010)

7. Wu, M., et al.: A small sphere and large margin approach for novelty detection using training data with outliers. IEEE Tran. on Pattern Anal. and Machine Intel. 31(11), 2088-2092 (2009)

8. Allwein, E.L., et al.: Reducing multiclass to binary: a unifying approach for margin classifiers. J. of Machine Learning Research 1, 113-141 (2001)

9. Dietterich, T.G., et al.: Solving multiclass learning problems via error-correcting output codes. J. of Artificial Intelligence Research 2, 263 (1995)

10. Fürnkranz, J.: Round robin classification. JMLR 2, 721-747 (2002)

11. Masulli, F., et al.: Comparing decomposition methods for classication. In: 4th Intl. Conf. on Knowledge-Based Intel. Engineering Systems \& Allied Technologies, pp. 788-791 (2000)

12. Rajan, S., et al.: An Empirical Comparison of Hierarchical vs. Two-Level Approaches to Multiclass Problems. In: Roli, F., Kittler, J., Windeatt, T. (eds.) MCS 2004. LNCS, vol. 3077, pp. 283-292. Springer, Heidelberg (2004)

13. Dietterich, T., et al.: Solving multiclass learning problem via error-correcting output codes. J. of Artificial Intel. Research 2, 263-286 (1995)

14. Mayoraz, E., et al.: On the decomposition of polychotomies into dichotomies. In: Proc. of the 14th Int. Conf. on Machine Learning, pp. 219-226 (1997)

15. Iannello, G., et al.: On the use of classification reliability for improving performance of the one-per-class decomposition method. Data \& Knowledge Eng. 68, 1398-1410 (2009)

16. Foggia, P., et al.: On Rejecting Unreliably Classified Patterns. In: Haindl, M., Kittler, J., Roli, F. (eds.) MCS 2007. LNCS, vol. 4472, pp. 282-291. Springer, Heidelberg (2007)

17. Cordella, L.P., et al.: Reliability parameters to improve combination strategies in multi-expert systems. Pattern Analysis \& Applications 2(3), 205-214 (1999)

18. Platt, J.: Probabilistic output for support vector machines and comparisons to regularize likelihood methods. Advanced in Large Margin Classifiers. MIT Press (2000)

19. Kanade, T., et al.: Comprehensive database for facial expression analysis. In: 4h IEEE Int. Conf. on Automatic Face and Gesture Recognition, pp. 46-53 (2000)

20. D'Ambrosio, R., et al.: Automatic Facial Expression Recognition Using Statistical-Like Moments. In: Maino, G., Foresti, G.L. (eds.) ICIAP 2011, Part I. LNCS, vol. 6978, pp. 585-594. Springer, Heidelberg (2011)

21. Soda, P., et al.: A multiple experts system for classifying fluorescence intensity in antinuclear autoantibodies analysis. Pattern Analysis \& Applications 12(3), 215-226 (2009)

22. Soda, P., et al.: Aggregation of classifiers for staining pattern recognition in antinuclear autoantibodies analysis. IEEE T Inf. Technol. B 13(3), 322-329 (2009)

23. Frank, A., et al.: UCI machine learning repository (2010) 